\title{
Prototype Development of a Conduction-Cooled LTS Pulse Coil for UPS-SMES
}

\author{
Toshiyuki Mito, Akifumi Kawagoe, Hirotaka Chikaraishi, Kagao Okumura, Ryo Abe, Tsutomu Henmi, \\ Ryuji Maekawa, Kazutaka Seo, Tomosumi Baba, Mitsuhiro Yokota, Yoshitaka Morita, Hideki Ogawa, \\ Kenji Yamauchi, Masataka Iwakuma, and Fumio Sumiyoshi
}

\begin{abstract}
We are planning to develop a 1 MW, 1 sec UPS-SMES for a protection from a momentary voltage drop and an instant power failure. As the first step, we have been developing a $100 \mathrm{~kJ}$ class prototype UPS-SMES, using a low temperature superconducting coil because of its better cost and performance over the high temperature superconducting coil. However, the difficulty to utilize a pool-boiling LTS pulse coil is the reliability of operation. To solve this problem, a conduction-cooled LTS pulse coil has been designed and fabricated as a key component of the UPS-SMES. The reduction of $\mathrm{AC}$ loss and high stability are required for the $\mathrm{SC}$ conductor for the conduction-cooled coil because of a limited cooling capacity. The $\mathrm{SC}$ conductor of a $\mathrm{NbTi} / \mathrm{Cu}$ compacted strand cable extruded with an aluminum is designed to have the anisotropic $\mathrm{AC}$ loss properties to minimize the coupling loss under the specified orientation of the time varying magnetic field. The coil was wound with a new twist-winding method in which the variation of twist angle of the conductor was controlled with the winding machine designed specifically for this purpose. The fabrication technique and performance of a conduction-cooled prototype LTS pulse coil are described.
\end{abstract}

Index Terms-Conduction cooled, momentary voltage drop, superconducting pulse coil, UPS-SMES.

\section{INTRODUCTION}

A momentary voltage drop and an instant power failure result in serious damage to production lines of an industrial plant. An uninterruptible power supply (UPS) with short time duration but large electric power capacity has been required as a suitable protection from those accidents. A Low Temperature Superconducting (LTS) pulse coil has excellent characteristics in the short-time energy extraction, which is adequate for a short-time UPS. Thus, we determined to utilize conductively cooled LTS pulse coil because of their higher reliability and easier operation than conventional cooling schemes such as a pool boiling or forced-cooling. Five-year project to develop a UPS-SMES with a capacity of $1 \mathrm{MW}$ and a short time duration of 1 second started in the 2002 fiscal year as one of the research promotion program of the New Energy and industrial

Manuscript received October 5, 2004. This work is supported in part by the grant of NEDO and the grant-in aid for Scientific Research (\#16206028).

T. Mito, H. Chikaraishi, R. Maekawa, K. Seo, T. Baba, M. Yokota, Y. Morita, H. Ogawa, and K. Yamauchi are with the National Institute for Fusion Science, Gifu 509-5292, Japan (e-mail: mito@nifs.ac.jp).

A. Kawagoe and F. Sumiyoshi are with the Kagoshima University, Kagoshima 890-0065, Japan.

K. Okumura is with the Technova Inc., Tokyo 100-0011, Japan.

$\mathrm{R}$. Abe is with the IDX Co., Tokyo 141-0022, Japan.

T. Henmi is with the Graduate University for Advanced Studies, Gifu 5095292, Japan.

M. Iwakuma is with the Kyushu University, Fukuoka 812-8581, Japan.

Digital Object Identifier 10.1109/TASC.2005.849339

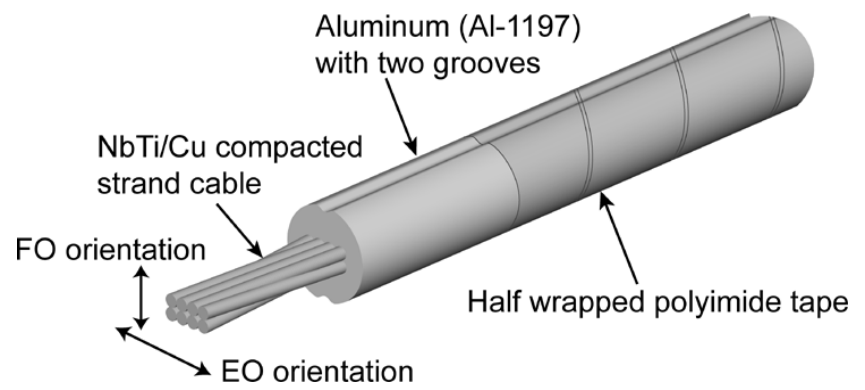

Fig. 1. Superconducting conductor for the LTS pulse coil.

technology Development Organization (NEDO) [1]. To confirm the feasibility of SMES with the LTS pulse coil, we have fabricated a prototype coil with stored energy of $100 \mathrm{~kJ}$. The paper describes a development of pulse coils with a new winding technique.

\section{Design of A CONDUCTION-CoOled LTS Pulse CoIL}

\section{A. Low AC Loss and High Stability SC Conductor}

A low AC loss and a high stability are required for the superconducting (SC) conductor of the conduction-cooled LTS pulse coil. The SC conductor of a NbTi/Cu compacted strand cable extruded with aluminum has been developed, whose configuration is shown in Fig. 1 and the parameters are listed in Table I. A NbTi conductor has advantages over $\mathrm{Nb}_{3} \mathrm{Sn}$ and/or HTS conductors because of low cost, easy handling and high commercial productivity

The conductor has the anisotropic coupling loss properties depending on the orientation of the time varying magnetic field. The coupling loss becomes the minimum when the time varying magnetic field is applied on the edge-on (EO) orientation to the compacted strand cable in the conductor. Therefore, the AC loss of the coil can be minimized as twisting the conductor during the winding process so that the $\mathrm{EO}$ orientation of the conductor coincides with the orientation of the magnetic field in the coil. An ordinal aluminum (Al-11 197) with a small residual resistivity ratio of 9.85 , which is extruded with a $\mathrm{NbTi} / \mathrm{Cu}$ compacted strand cable, is adapted not for a stabilizer but for a supporting guide of the twist winding to minimize the AC loss. The aluminum is also important as a heat sink to suppress temperature rise during the pulse operation of the conduction-cooled coil. The conductor has two grooves on the round shape cross section as shown in Fig. 1 to detect the angle of the compacted strand cable inside the conductor.

The estimated inter-strand contact resistance in the compacted strand cable of the conductor is very small about 
TABLE I

SPECIFICATIONS OF THE SC CONDUCTOR

\begin{tabular}{ll}
\hline \hline Conductor type & $\begin{array}{l}\text { Aluminum coated NbTi/Cu compacted } \\
\text { strand cable }\end{array}$ \\
Conductor diameter & $5.8 \mathrm{~mm}$ \\
Cross section shape & Round with two grooves \\
Operational current & $1000 \mathrm{~A}$ \\
Critical current & $3740 \mathrm{~A} \mathrm{@} 5 \mathrm{~T}, 4.2 \mathrm{~K}$ \\
Insulation & $25 \mu \mathrm{m}$ thick $\times 15 \mathrm{~mm}$ wide \\
Outer diameter with insulation & Half wrap winding of Polyimide tape \\
Tensile strength & $5.9 \mathrm{~mm}$ \\
0.2\% Yield strength & $96 \mathrm{MPa}$ \\
Weight & $49 \mathrm{Mpa}$ \\
Coupling time constant of AC & $94.3 \mathrm{~kg} / \mathrm{km}$ \\
loss: $\mathrm{n} \tau$ & $82 \mathrm{msec}$ for face-on (FO) orientation \\
Compacted strand cable & $10 \mathrm{msec}$ for edge-on (EO) orientation \\
$\quad$ & \\
Number of strands & 8 \\
Strand diameter & $0.823 \mathrm{~mm}$ \\
Dimension & $1.55 \times 3.36 \mathrm{~mm}$ \\
Aluminum coating & \\
$\quad$ Material & $\mathrm{Al}-1197$ \\
RRR & 9.85 \\
\hline \hline
\end{tabular}

a)

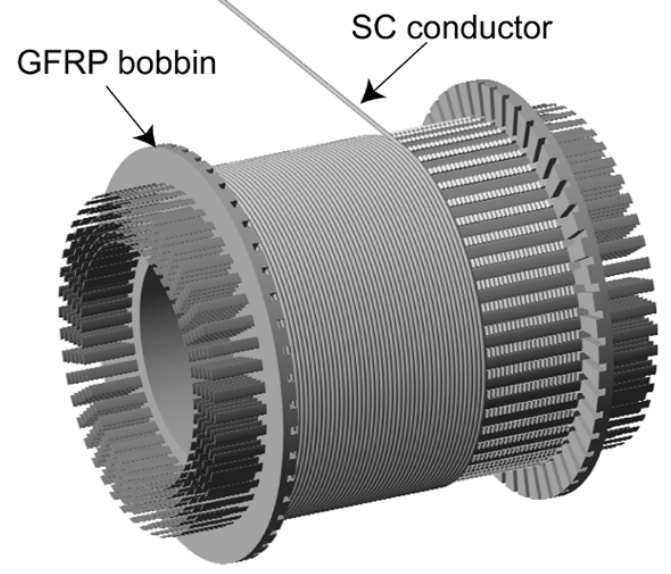

b)

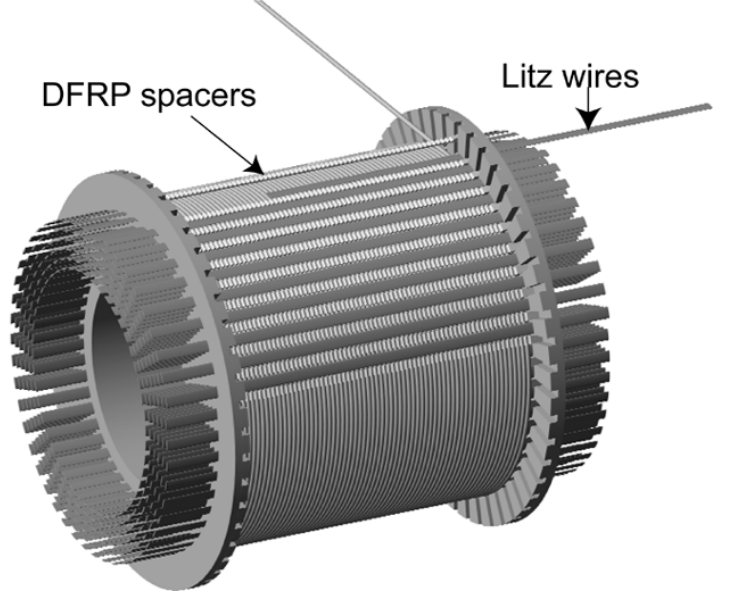

Fig. 2. Winding procedure of the prototype LTS pulse coil, a) winding each layer, b) setting DFRP spacers and inserting Litz wires after the winding of each layer.

$10^{-12} \Omega \mathrm{m}^{2}$, which ensures good current redistribution characteristics and a high stability of the conductor [2].
TABLE II

SPECIFICATIONS OF THE PROTOTYPE LTS PULSE COIL

\begin{tabular}{ll}
\hline \hline Cooling method & $\begin{array}{l}\text { Conduction cooling with Litz wires } \\
\text { Coil shape }\end{array}$ \\
Winding method & $\begin{array}{l}\text { Solenoid } \\
\text { Twist winding to minimize AC loss }\end{array}$ \\
Dimension of the coil windings & \\
$\quad$ Inner diameter: 2a1 & $0.305 \mathrm{~m}$ \\
$\quad$ Outer diameter: 2a2 & $0.509 \mathrm{~m}$ \\
$\quad$ Length: 2L & $0.402 \mathrm{~m}$ \\
Number of turns per 1 layer & 67 \\
Number of layers & 14 \\
Total turn number & 938 \\
Total length of conductor & $1.20 \mathrm{~km}$ \\
Coil inductance & $0.20 \mathrm{H}$ \\
Maximum magnetic field & $2.2 \mathrm{~T}$ \\
Magnetic stored energy & $100 \mathrm{~kJ}$ \\
Start operating current & $1000 \mathrm{~A}$ \\
Stop current after 1 sec discharge & $707 \mathrm{~A}$ \\
Maximum discharge energy & $50 \mathrm{~kJ}$ \\
Litz wies for conduction cooling & Braided wires of insulated copper \\
Strands \\
Total coil weight & $4,774 \times 2=9,548 \mathrm{~mm}^{2}$ \\
Superconducting conductor & $400 \mathrm{~kg}$ \\
GFRP winding bobbin & $113 \mathrm{~kg}$ \\
DFRP spacers & $70 \mathrm{~kg}$ \\
Litz wires & $40 \mathrm{~kg}$ \\
Epoxy resin & $115 \mathrm{~kg}$ \\
Others & $39 \mathrm{~kg}$ \\
\hline \hline & $23 \mathrm{~kg}$ \\
\hline
\end{tabular}

\section{B. Configuration of Prototype Conduction-Cooled Pulse Coil}

The winding procedure of the prototype conduction-cooled LTS pulse coil is shown in Fig. 2 and its parameters are listed in Table II. The coil shape is a single solenoid of 67 turns $\times$ 14 layers wound on the GFRP bobbin. The Dyneema FRP (DFRP) spacers and the Litz wires (braided wires of insulated copper strands) are inserted in each layer as shown in Fig. 2(b). The DFRP spacers with a thickness of $10 \mathrm{~mm}$ and a length of $428 \mathrm{~mm}$ have been machined with the semi-circle grooves so that the round conductor can be settled at the exact winding position. The transition of the windings from a layer to the next layer can be done easily by changing the position of the semi-circle grooves at both ends of the DFRP spacers according to their circumferential settled position.

The DFRP spacers have a good thermal conductivity along with Dyneema filaments, which enhance the heat transfer from layer to layer in the windings. On the other hand, the Litz wires increase the heat transfer from turn to turn in the windings and enable conduction cooling of the coil by attaching the end of the Litz wires directly to the cold heads of the cryocoolers.

\section{Construction of the Prototype Conduction-Cooled PULSE COIL}

\section{A. Development of the New Winding Technique}

A difficult point to fabricate a conduction-cooled pulse coil is how to twist the $\mathrm{SC}$ conductor according to the orientation of magnetic field in the winding process. It is also necessary to establish a fabrication technique which is simple and suitable for mass production of UPS-SMES coils. Therefore, we have developed an automatic winding machine which can control a twisting angle of the conductor according to the winding position as shown in Fig. 3. The twisted angle of the conductor 


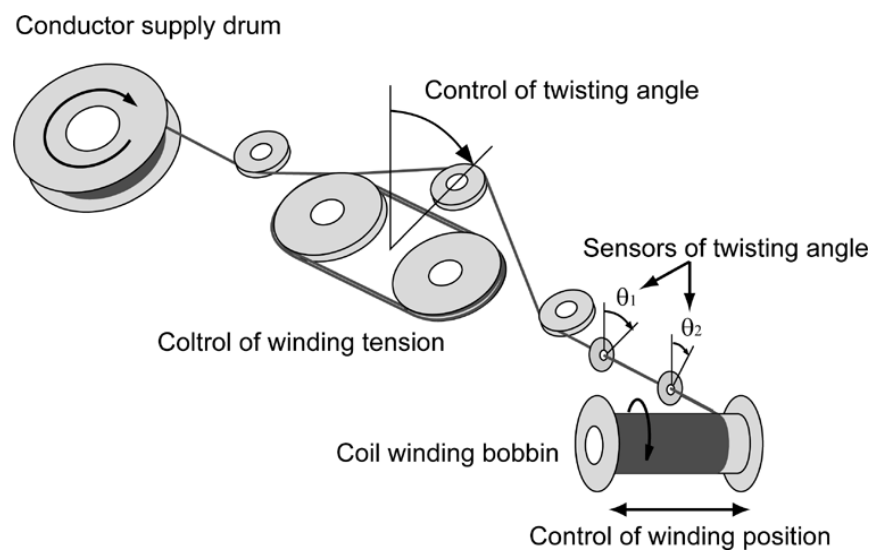

Fig. 3. Principle operation of a twist winding machine.

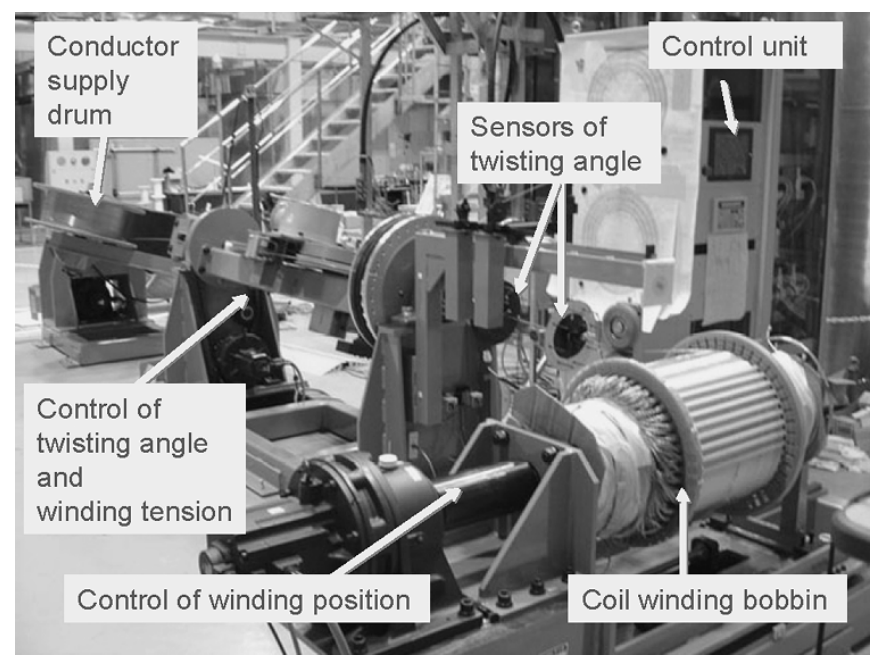

Fig. 4. Winding procedure of the prototype coil by the developed twist winding machine.

can be measured by two angle sensors installed just before the winding bobbin. The angle sensor has a proximity sensor which rotates around the conductor and detects the groove on the conductor. Then the twist angle of the conductor at the position of the winding bobbin is extrapolated from the measured values of two angle sensors. Fig. 4 shows the developed winding machine, which can control the winding tension, the axial position of the winding bobbin and the twist angle of the conductor according to the programmed table, and Fig. 4 also shows the winding procedure of the prototype coil.

\section{B. Winding Procedure of the Prototype Coil}

Fig. 5 shows the prototype conduction cooled coil during the winding process. The coil was wound in two weeks at the pace of 1 layer/day. The twisting angle of the conductor was measured every $1 / 4$ turn in the center part and every $1 / 12$ turn in the end part of the coil and was used for the feedback control of twisting angle. Fig. 6 shows the measured errors of the twisting angle of the conductor at the 12th layer of the windings as an example. The errors were within \pm 10 degrees and their root mean square is 3.1 degrees. The root mean square (RMS) of the twisting angle errors for each layer of the windings were summarized in Table III. The RMS errors were larger then 5 degrees

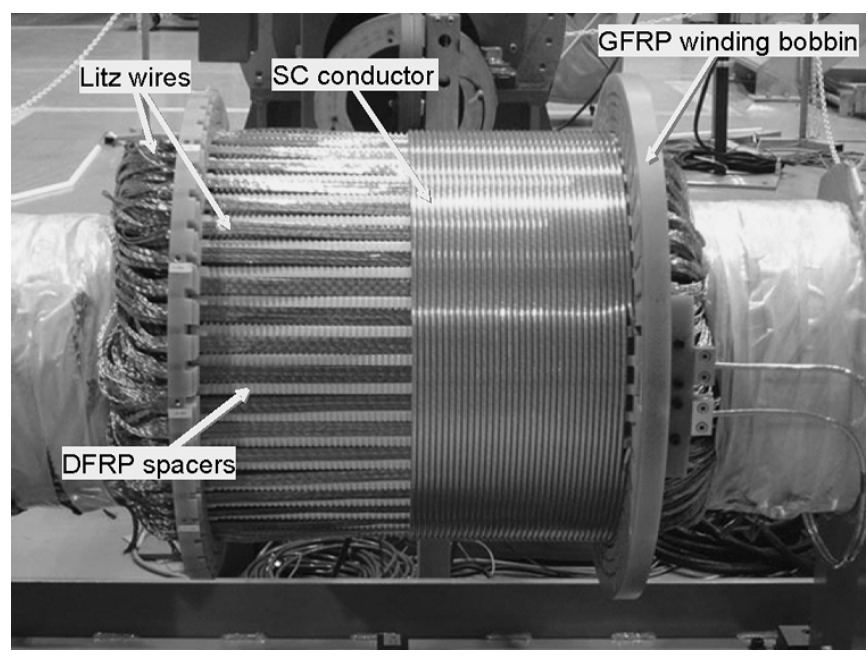

Fig. 5. Prototype conduction-cooled coil during the winding process.

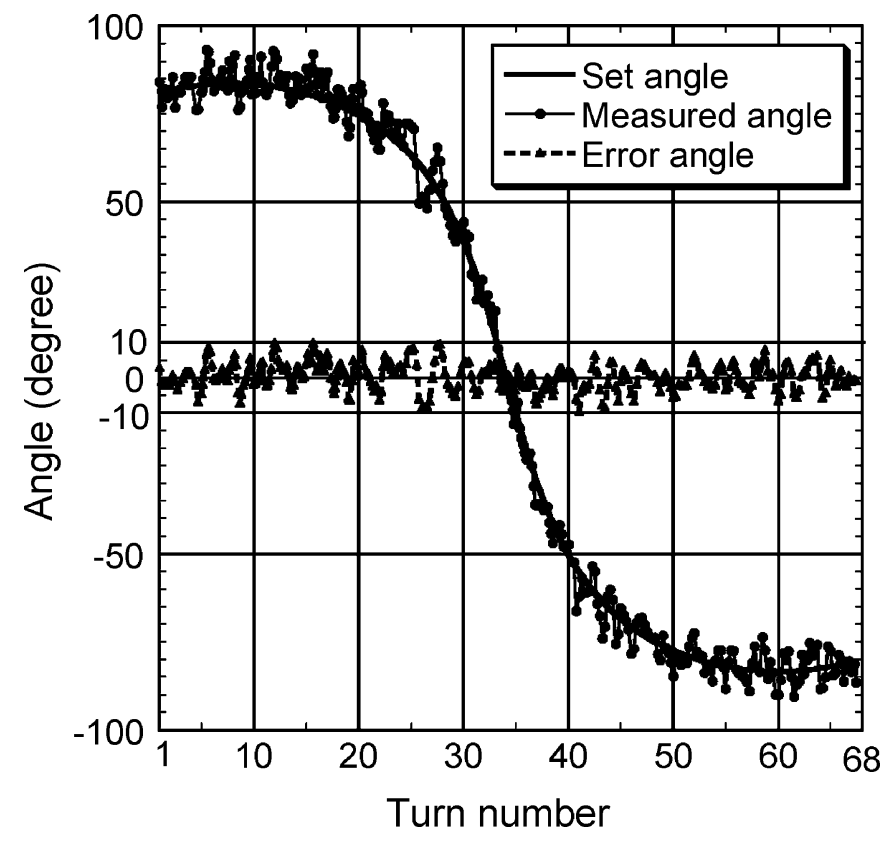

Fig. 6. Measured errors of the twisting angle of the conductor at 12th layer of the windings.

TABLE III

TWISTING ANGLE ERRORS OF EACH LAYER OF THE WINDINGS

\begin{tabular}{lc|cc}
\hline \hline $\begin{array}{l}\text { Layer } \\
\text { No. }\end{array}$ & RMS angle error (deg) & $\begin{array}{l}\text { Layer } \\
\text { No. }\end{array}$ & RMS angle error (derg) \\
\hline 1 & 4.3 & 8 & 9.3 \\
2 & 5.7 & 9 & 3.5 \\
3 & 6.0 & 10 & 3.5 \\
4 & 5.4 & 11 & 3.8 \\
5 & 5.8 & 12 & 3.1 \\
6 & 5.6 & 13 & 3.9 \\
7 & 5.9 & 14 & 3.9 \\
\hline \hline
\end{tabular}

until no. 8 layer of the windings. However after the improvement of the twisting angle control, we have succeeded to develop the twist winding technique with the RMS error less than 5 degrees, which is enough for suppressing AC losses of the coil [2]. 


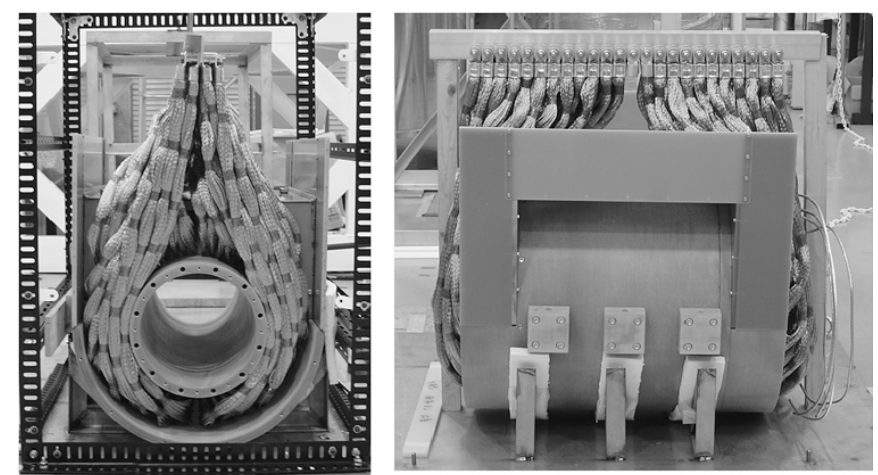

Fig. 7. Prototype coil with the Litz wires for conduction cooling just before epoxy impregnation.

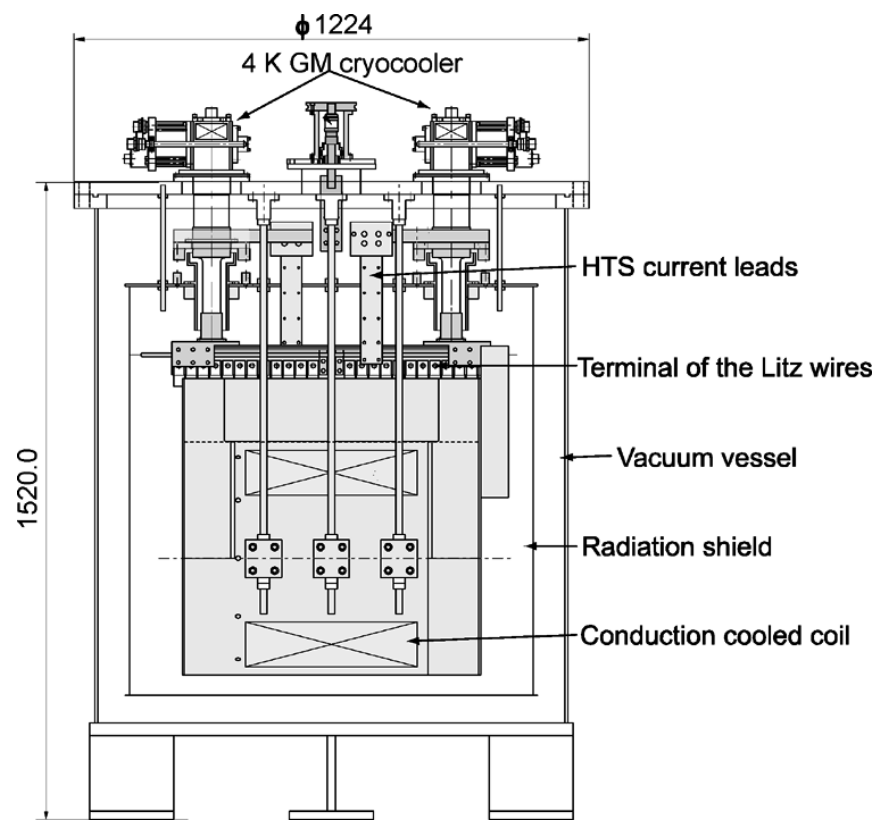

Fig. 8. Cryostat for cooling and excitation tests of the prototype coil.

\section{Experimental Setup}

Fig. 7 shows the prototype coil after winding and before epoxy impregnation. The ends of the Litz wires were bundled and terminated with solderless contacts. Fig. 8 shows experimental setup for cooling and excitation test. The coil is covered with the radiation shield cooled by the 1st stages of two cryocoolers, and is installed into the vacuum vessel. The terminals of the Litz wires were attached to the cold head of two cryocoolers. The heat loads to the conduction cooled coil and cooling capacity of GM cryocoolers are listed in Table IV. The total copper cross section of the Litz wires is $0.009548 \mathrm{~m}^{2}$, and the average length of the Litz wires from the coil to the 2 nd stage of the cryocooler is $1.10 \mathrm{~m}$. If the $2 \mathrm{nd}$ stage temperature of the cryocooler is estimated to be $4.0 \mathrm{~K}$ and the heat input
TABLE IV

HeAt LoAds of THE PRototype Conduction CoOled CoIL

\begin{tabular}{|c|c|}
\hline \multicolumn{2}{|c|}{ Cooling capacity of two GM cryocoolers } \\
\hline $\begin{array}{l}\text { Cooling capacity of } 1^{\text {st }} \text { stage }(@ 50 \mathrm{~K}) \\
\text { Cooling capacity of } 2^{\text {nd }} \text { stage }(@ 4 \mathrm{~K})\end{array}$ & $\begin{array}{l}120 \mathrm{~W} \\
3 \mathrm{~W} \\
\end{array}$ \\
\hline \multicolumn{2}{|c|}{ Heat loads@50 K } \\
\hline Copper current leads (1000 A) & $84.0 \mathrm{~W}$ \\
\hline Radiation & $8.0 \mathrm{~W}$ \\
\hline Supports & $1.4 \mathrm{~W}$ \\
\hline Piping, etc. & $0.4 \mathrm{~W}$ \\
\hline Total & $93.8 \mathrm{~W}$ \\
\hline \multicolumn{2}{|c|}{ Heat loads@4K } \\
\hline$\overline{\text { HTS current leads (1000 A) }}$ & $1.0 \mathrm{~W}$ \\
\hline Radiation & $0.6 \mathrm{~W}$ \\
\hline Supports & $0.1 \mathrm{~W}$ \\
\hline Piping, etc. & $0.1 \mathrm{~W}$ \\
\hline Total & $1.8 \mathrm{~W}$ \\
\hline
\end{tabular}

to the coil is $1.8 \mathrm{~W}$, the temperature of the coil can be kept at $4.4 \mathrm{~K}$ during the standby operation of the UPS-SMES.

The performance of the conduction-cooled pulse coil is determined by the temperature margin during pulse operation. The temperature rise of the prototype coil after $1 \mathrm{sec}$ discharge is estimated as $6.7 \mathrm{~K}$ with its current sharing temperature of $8.2 \mathrm{~K}$, assuming an adiabatic condition in which the heat transfer from the SC conductor to the DFRP spacers and other winding components was neglected. The detailed analysis including the heat transfer during the pulse operation has been carried out [3] and the temperature margin was estimated to increase from $1.5 \mathrm{~K}$ to $2.5 \mathrm{~K}$. We will perform the verification tests of the prototype coil by the end of 2004 .

\section{SUMMARY}

We have been developing a conduction-cooled LTS pulse coil as the most reliable and cost effective superconducting coil for the UPS-SMES. The construction of a $100 \mathrm{~kJ}$ prototype coil has been successfully achieved by developing the special winding techniques for the conduction-cooled LTS pulse coil. For the next step, $1 \mathrm{MJ}$ conduction-cooled LTS pulse coils are planned to be fabricated during the 2004 fiscal years. In the 2005 fiscal year, the performance tests of the $1 \mathrm{MJ}$ coils will be conducted, and the long-term field test of the UPS-SMES using the $1 \mathrm{MJ}$ LTS pulse coils is planned in the 2006 fiscal year.

\section{REFERENCES}

[1] T. Mito et al., "Development of UPS-SMES as a protection from momentary voltage drop," IEEE Trans. on Appl. Super, vol. 14, no. 2, pp. 721-726, 2004.

[2] A. Kawagoe et al., "Development of conduction cooled LTS pulse coils for $100 \mathrm{~kJ}$ class UPS-SMES as a protection from momentary voltage drop," IEEE Trans. on Appl. Super., vol. 14, no. 2, pp. 727-730, 2004.

[3] - "Stability evaluation of a conduction-cooled prototype LTS pulse coil for UPS-SMES," presented at the ASC 2004, 1LG05. 